\title{
Observation of Plasmarons in Quasi-Free-Standing Doped Graphene
}

\author{
Aaron Bostwick, ${ }^{1}$ Florian Speck, ${ }^{2}$ Thomas Seyller, ${ }^{2}$ Karsten Horn, ${ }^{3}$ Marco Polini, ${ }^{* 4}$ Reza Asgari, ${ }^{* 5}$ Allan H. \\ MacDonald, ${ }^{6}$ Eli Rotenberg ${ }^{\dagger 1}$
}

\begin{abstract}
${ }^{1}$ E. O. Lawrence Berkeley Laboratory, Advanced Light Source, MS6-2100, Berkeley, CA 94720
${ }^{2}$ Lehrstuhl für Technische Physik, Universität Erlangen-Nürnberg, Erwin-Rommel-Str. 1, 91058 Erlangen, Germany

${ }^{3}$ Dept. of Molecular Physics, Fritz-Haber-Institut der Max-Planck-Gesellschaft, Faradayweg 4-6, 14195 Berlin, Germany

${ }^{4}$ NEST, Istituto Nanoscienze-CNR and Scuola Normale Superiore, I-56126 Pisa, Italy"

${ }^{5}$ School of Physics, Institute for Research in Fundamental Sciences (IPM), Tehran 19395-5531, Iran

${ }^{6}$ Dept. of Physics, Univ. of Texas at Austin, 1 University Station C1600, Austin TX 78712

*These authors contributed equally to this work.

†To whom correspondence should be addressed
\end{abstract}

A hallmark of graphene is its unusual conical band structure that leads to a zero-energy band gap at a single Dirac crossing point. By measuring the spectral function of charge carriers in quasi-free-standing graphene with angle-resolved photoemission spectroscopy, we show that at finite doping the well-known linear Dirac spectrum does not provide a full description of the charge-carrying excitations. We observe composite "plasmaron" particles, which are bound states of charge carriers with plasmons, the density oscillations of the graphene electron gas. The Dirac crossing point is resolved into three crossings: the first between pure charge bands, the second between pure plasmaron bands, and the third a ring-shaped crossing between charge and plasmaron bands.

Electrons in metals and semiconductors undergo many complex interactions, and most theoretical treatments make use of the quasiparticle approximation, in which independent electrons are replaced by electron- and holelike quasiparticles interacting through a dynamically screened Coulomb force. The details of the screening are determined by the valence band structure, but the band energies are modified by the screened interactions. A complex self-energy function describes the energy and lifetime renormalization of the band structure resulting from this interplay.

Bohm and Pines $(l)$ accounted for the short-range interactions between quasiparticles through the creation of a polarization cloud formed of virtual electron-hole pairs around each charge carrier, screening each from its neighbors. The long-range interactions manifest themselves through plasmons, collective charge density oscillations of the electron gas that can propagate through the medium with their own band-dispersion relation. These plasmons can in turn interact with the charges, leading to strong self-energy effects. Lundqvist predicted the presence of new composite particles called plasmarons formed by the coupling of the elementary charges with plasmons (2). Their distinct energy bands should be observable using angle-resolved photoemission spectroscopy (ARPES), but so far have only been observed by optical $(3,4)$ and tunneling spectroscopies (5), which probe the altered density of states.

Understanding the coupling between electrons and plasmons is important because of new "plasmonic" devices proposed to merge photonics and electronics. Graphene in particular has been proposed as a promising candidate for such devices (6-8). Plasmarons have been predicted to occur in graphene and to be observable in ARPES $(9,10)$, yet their detailed dispersion and interaction with defects remain unknown. Here we present a 

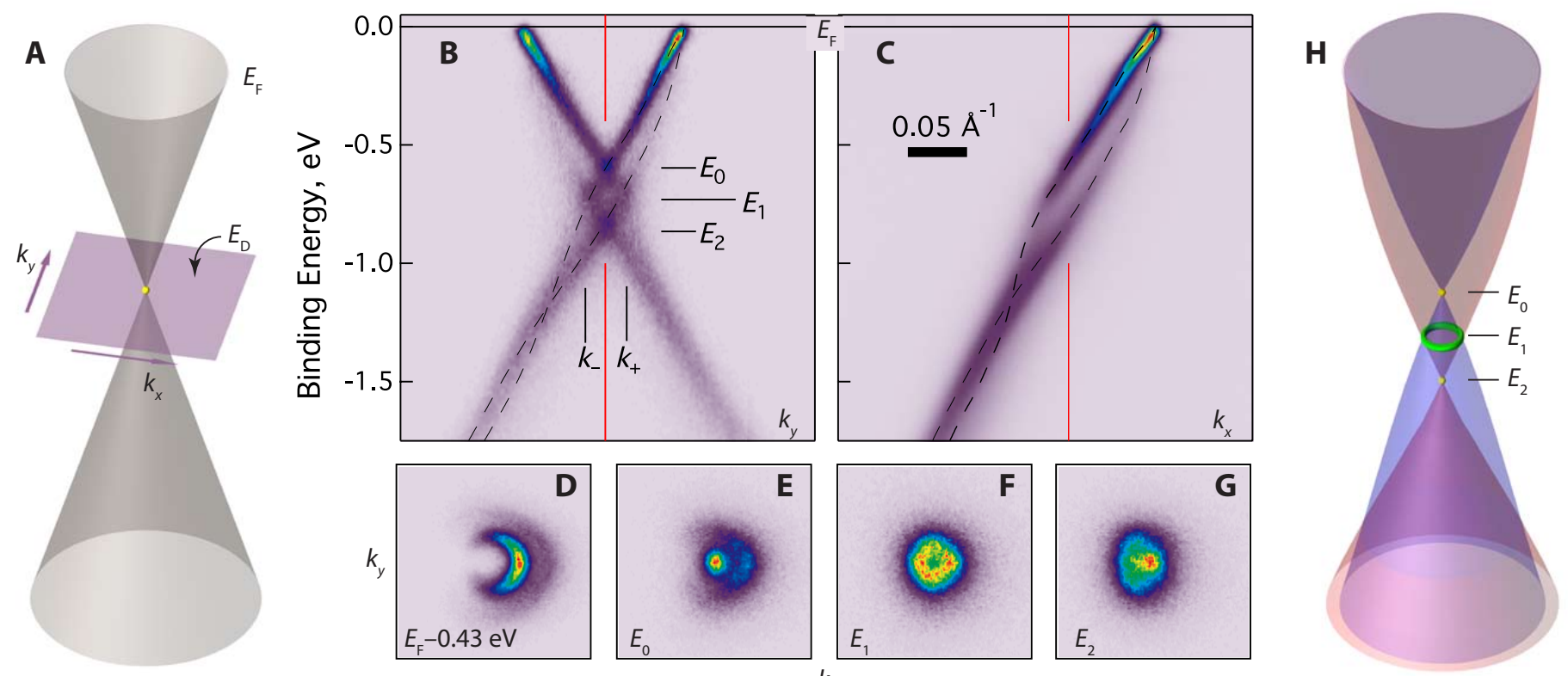

Fig. 1. A The Dirac energy spectrum of graphene in a non-interacting, single-particle picture. B-C Experimental spectral functions of doped graphene perpendicular and parallel to the $\Gamma K$ direction of the graphene Brillouin zone in momentum space. The dashed lines are guides to the dispersion of the observed hole and plasmaron bands. The red lines are at $k=0$ (the $\mathrm{K}$ point of the graphene Brillouin Zone). D-G constant-energy cuts of the spectral function reveal the chiral character of the hole and plasmaron bands, and the cylindrical symmetry of the bands near the Dirac crossing region. $\mathbf{H}$ the Dirac spectrum in the presence of interactions is reconstructed into two Dirac crossing points at energies $E_{0}$ and $E_{2}$, and a "Dirac ring" at $E_{1}$. The sample used for parts B-G was doped to a level $n=1.7 \times 10^{13} \mathrm{~cm}^{-2}$.

systematic ARPES study of doped graphene, which reveals details of the plasmaron band structure.

In a non-interacting picture, the dispersion relation between its carriers' energy $\omega$ and their momentum $\mathbf{k}$ is expressed by the equation $\omega_{\mathrm{b}}=v_{\mathrm{F}} k$ where $v_{\mathrm{F}}$ is the Fermi velocity of the carriers (11-14). Here the subscript " $\mathrm{b}$ " reminds us that this is the "bare" dispersion. The bands cross at a single point in momentum space $(k=0)$, the so-called Dirac crossing energy $E_{\mathrm{D}}$ as shown in Fig. 1A. Because of the symmetry of graphene's honeycomb lattice, the spectrum is gapless, and the fundamental particles are effectively massless Dirac Fermions. This gives rise to a chiral degree of freedom represented by a pseudospin vector (11-14).

We now show that in reality the topology of the bands near the Dirac crossing differs significantly from this well-known picture. Fig. 1B shows the energy band structure of $n$-doped "quasi-free-standing" graphene, grown epitaxially on H-terminated $\mathrm{SiC}$ by a newly described method $(15,16)$, chemically doped using potassium atoms (17), and measured with ARPES. Instead of a pair of bands crossing at the Dirac energy $E_{\mathrm{D}}$, we find four bands, two of them highlighted by dashed lines (the other two are equivalent by reflection across $k=0$ ). The data reveal a rearrangement of states around $E_{\mathrm{D}}$ from a single point crossing into a diamond-like shape, characterized by three energies $E_{0}, E_{1}, E_{2}$ at its apex, middle, and bottom, and two momenta $k_{ \pm}$defining its width. Acquiring the spectrum in a different geometry we can determine the band structure projected onto a single pseudospin orientation $(18,19)$, as shown in Fig. 1C. This shows that the two highlighted bands have the same chirality. This is confirmed by examining constant-energy cuts of the spectral function (Fig. 1D-G) which show two bands having identical intensity distributions about the center. The crossing of bands of opposite 
chirality implies the existence of two Dirac crossings at $E_{0}$ and $E_{2}$, (Fig. 1E and G) and a ring-like crossing at energy $E_{1}$, which is resolved in Fig. 1F.

In summary, the single-particle picture in Fig. 1A fails to account for the self-energy arising from interactions between a single charge carrier and the sea of electrons forming a gas in graphene, and these self-energy effects directly affect the spectral function measured by ARPES. These data can be better explained with a different model of the electronic structure, as illustrated in Fig. $1 \mathrm{H}$.

In photoemission, a soft x-ray photon promotes an electron from the occupied bands into a free state that is detected by an electron spectrometer, leaving a hole behind. The renormalization of the photohole's lifetime and mass alter the single-particle spectral function $A(\mathbf{k}, \omega)$ of the excited photohole measured by ARPES. Here $\mathbf{k}$ is the photohole momentum and $\omega<0$ its binding energy. The spectral function $A(\mathbf{k}, \omega)$ is related to the complex self-energy $\Sigma(\mathbf{k}, \omega)$ and the bare band $\omega_{\mathrm{b}}$ through the equation

$$
\mathrm{A}(\mathrm{k}, \omega)=\frac{\pi^{-1}|\operatorname{Im} \Sigma(\mathrm{k}, \omega)|}{\left(\omega-\omega_{\mathrm{b}}(\mathrm{k})-\operatorname{Re} \Sigma(\mathrm{k}, \omega)\right)^{2}+(\operatorname{Im} \Sigma(\mathrm{k}, \omega))^{2}}
$$

When the first term in the denominator is zero, a resonance is found in $A(\mathbf{k}, \omega)$ that reflects the existence of quasiparticles having a finite lifetime and an energy only slightly shifted from $\omega_{b}(\mathbf{k})$. The self-energy arising from short range interactions (mediated by $e-h$ pair generation) is typically small in metals and the lifetime decreases smoothly as the hole increases in energy, so that the band dispersion is only slightly different from the bare band.

In contrast, the full self-energy function $\Sigma(\mathbf{k}, \omega)$ can have a more complicated energy-dependence leading to multiple resonances at a given $\mathbf{k}$. When this occurs it signals the emergence of new charged entities that can propagate with well-defined momenta. In one-dimensional metals, for example, this can lead to breakup of a hole into separately observable spin- and charge-carrying bands, through so-called spin-charge separation (20). For two or more dimensions new composite particles such as polarons (hole + phonon) (21) or Kondo resonances (localized hole + screening cloud) (22) may be observed. In like manner, for graphene, we attribute the lower band (towards greater binding energy) in Fig. 1 to a distinct quasiparticle reflecting the formation of plasmarons (2), composite particles consisting (at a particular momentum $\mathbf{k}$ ) of holes with momentum $\mathbf{k}+\mathbf{q}$ strongly coupled to plasmons of momentum $-\mathbf{q}$.

This new plasmaronic quasiparticle appears at greater binding energy because of the extra energy cost of creating a plasmon with a hole, which then interact to form the plasmaron. Because the bare plasmon and bare hole energies depend on momentum, this description suggests a broad distribution of states towards higher binding energy, not a discrete quasiparticle. The sharpness of the plasmaronic quasiparticle arises for two reasons: first, because scattering a hole from a plasmon requires conservation of pseudospin as well as momentum, which favors $\mathbf{k}$ and $\mathbf{q}$ to be parallel. The excitation spectrum thus becomes effectively onedimensional, strengthening the interactions. Secondly, only plasmons and holes with the same group velocity are likely to form plasmarons (23), further reducing the phase space for hole-plasmon interactions. 


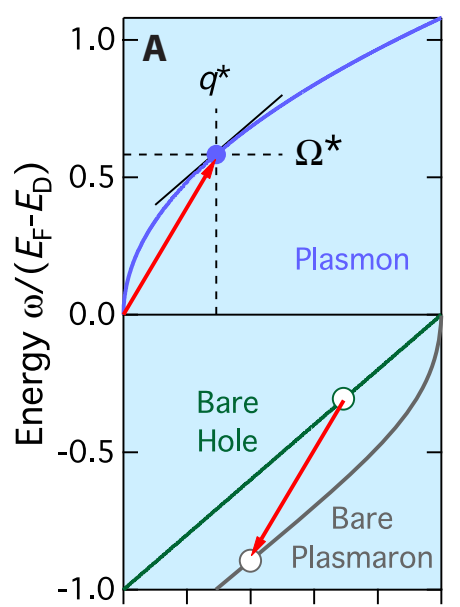

$\begin{array}{llllllllll}0.0 & 0.2 & 0.4 & 0.6 & 0.8 & 1.0\end{array}$

Momentum $k$ or $q, \times k_{\mathrm{F}}{ }^{-1}$
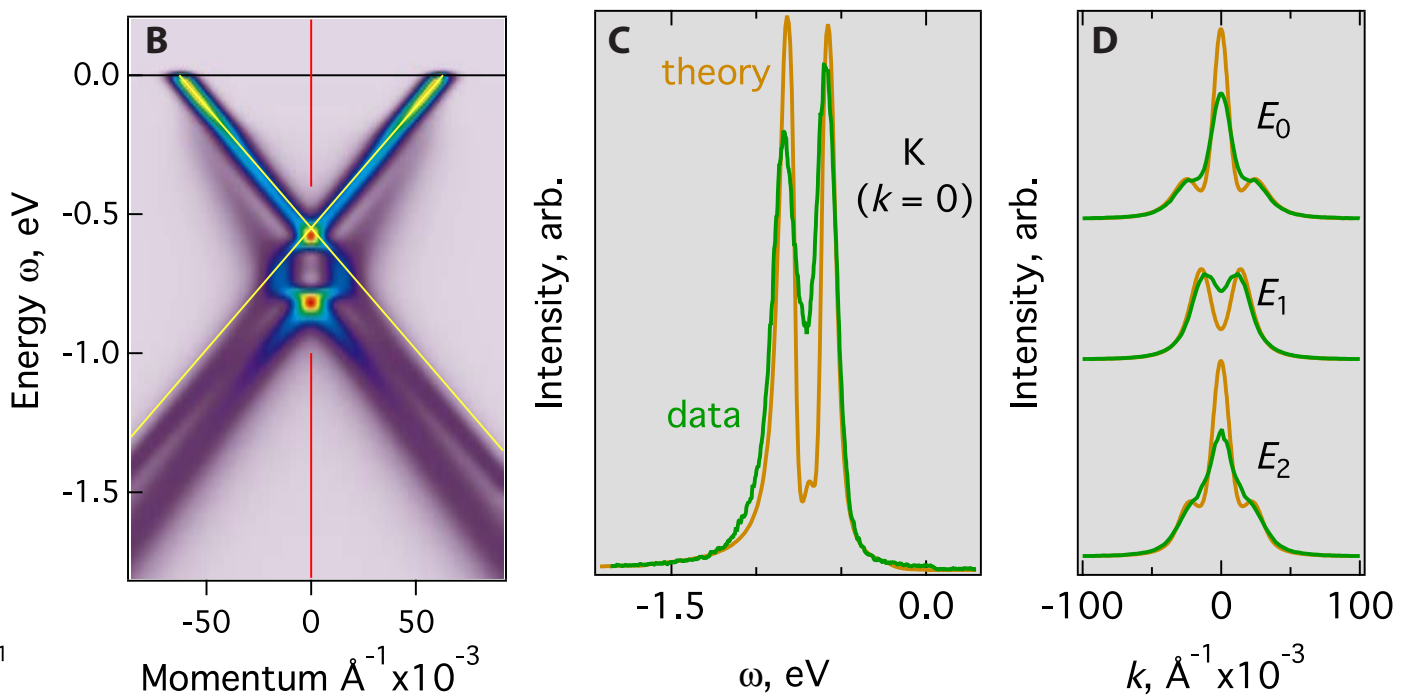

$\omega, \mathrm{eV}$

$k, \AA^{-1} \times 10^{-3}$

Fig. 2. A Comparison of (upper) plasmon dispersion function $\Omega(q)$ and (lower) bare hole and bare plasmaron quasiparticle dispersions. The red arrow, specified by velocity matching conditions (see text) defines the shift between plasmaron and hole bands in a simple kinematic description where the hole-plasmon binding is ignored. B The predicted spectral function along two orthogonal cuts according to $G_{0} W$-RPA theory, together with a phenomenological contribution from phonon scattering and instrumental broadening. The yellow lines indicate the bare band structure in the absence of interactions. C-D Comparison of the predicted and experimental spectra along different cuts of constant momentum or energy. In $\mathbf{D}$, the experimental cuts have been averaged over all azimuthal directions about $k=0$.

The kinematics of the "bare" plasmaronic band formation-that is, the formation of the plasmon plus hole without considering their mutual binding interaction-is shown in Fig. 2A. Comparing the plasmon dispersion function $\Omega(q)(10,24,25)$ to the bare band structure $\omega_{\mathrm{b}}(k)$, we see that while all holes have the same group velocity (given by the slope of the linear band), only one characteristic plasmon at a single momentum $q^{*}$, and energy $\Omega^{*}$ (indicated by the red arrow) has the same group velocity as all of the holes and therefore is favored to form the plasmaronic quasiparticle when their interaction is considered. Except near $E_{\mathrm{F}}$, the bare plasmaronic band is offset from the bare hole band by approximately these momentum and energy transfers $q^{*}$, and energy $\Omega^{*}$. Near $E_{\mathrm{F}}$, the kinematic restrictions reduce the possible energies and momenta of the interacting plasmon, causing a merging of a much-dampened plasmaron towards the hole band as $k \rightarrow k_{\mathrm{F}}$.

This simple model qualitatively predicts the position and shape of the bare plasmaronic band, but because it neglects the plasmon-hole binding, it underestimates the separation of the plasmaron and hole bands by almost a factor of 2. The actual shift is better represented by detailed calculations of the graphene spectral function Eq. (1) within the $G_{0} W$ approximation $(9,10)$. This approach approximates the self-energy to lowest order by a renormalized Green's function $G$ in which the self-energy is expanded to first order in the dynamically screened Coulomb interaction $W$ computed within the random phase approximation (RPA). An additional phenomenological self-energy arising from electron-phonon coupling is incorporated to account for the wellknown band sharpening and kink near $E_{\mathrm{F}}-180 \mathrm{meV}(17)$. The computed spectral function is broadened to account for the instrumental resolutions $\left(0.025 \mathrm{eV}\right.$ and $\left.0.01 \AA^{-1}\right)$. Fig. $2 \mathrm{~B}$ shows the resulting spectral function, which is in good qualitative agreement with the experimental data in Figs. 1B,C. These calculations agree well with our observation that the single Dirac crossing at $E_{\mathrm{D}}$ (indicated by the yellow, bare bands) is reconstructed 

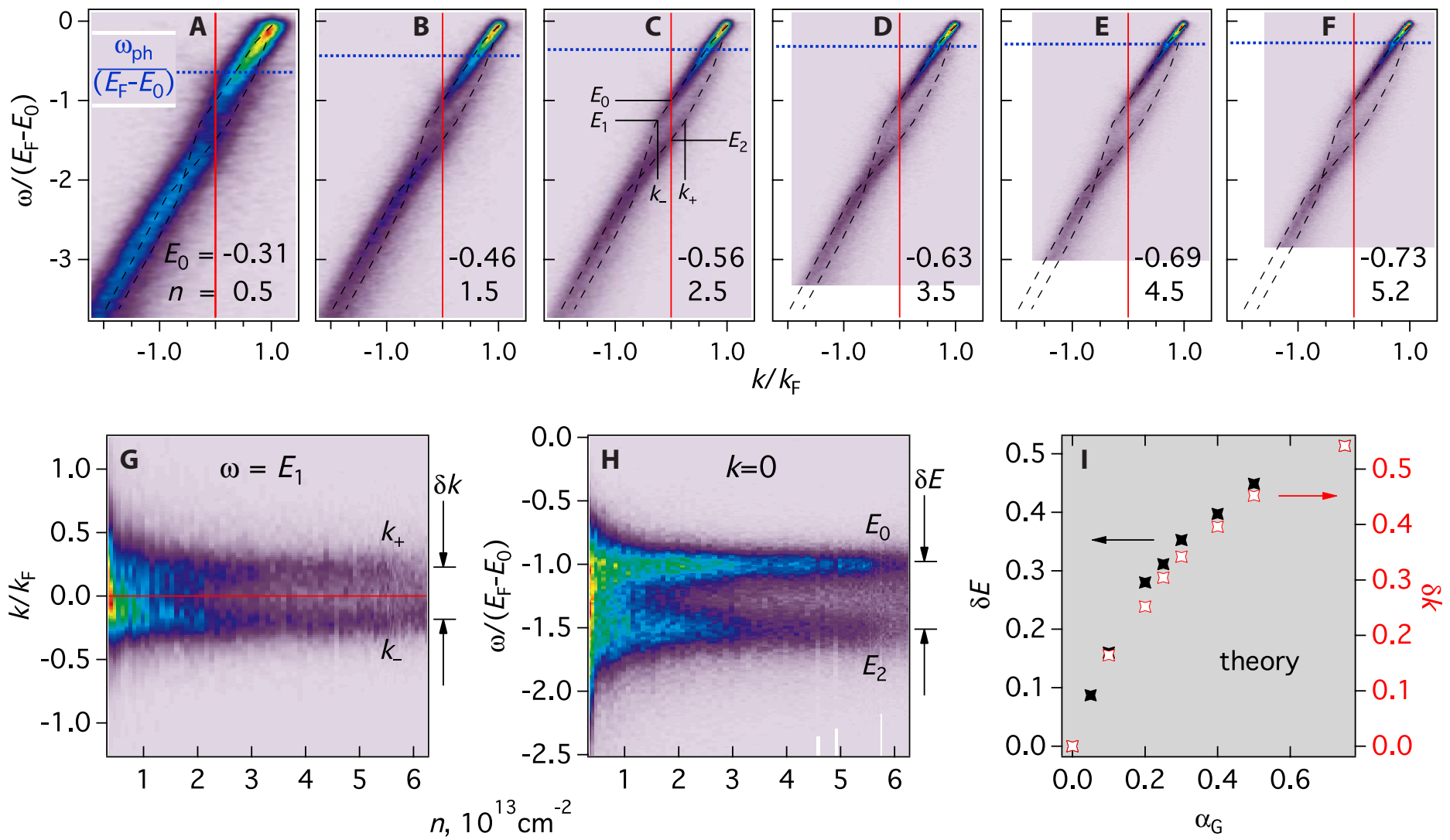

Fig. 3. The doping dependence of the graphene spectral function with energy $\omega$ and momentum $k$ scaled to $\left(E_{\mathrm{F}}-E_{0}\right)$ and $k_{F}$, resp. A-F. A sequence of bands acquired along the $\Gamma K$ direction of the graphene Brillouin Zone as a function of doping $n$ (and hence Dirac energy $E_{\mathrm{D}} \approx E_{0}$ ). The dashed lines are a guide to the eye and are the same in each plot. The dotted lines represent the scaled phonon energy scale $\omega_{\mathrm{ph}}$. Values of $E_{0}$ and $n$ are in units of $\mathrm{eV}$ and $10^{13} \mathrm{~cm}^{-2}$, resp. G-H constant energy and momentum cuts through the center of the diamond at the Dirac region, acquired for a series of 82 different dopings, after scaling to $k_{F}$ and $\left(E_{\mathrm{F}}-E_{0}\right)$. In all panels, the red lines represent $k / k_{\mathrm{F}}=0$. I The predicted variation of the horizontal and vertical dimensions $\delta k, \delta E$ of the diamond at $E_{\mathrm{D}} v s$. coupling constant $\alpha_{G}$.

into a diamond-shaped feature with apex energy $E_{0} \approx E_{\mathrm{D}}$.

These calculations describe the spectral function around the renormalized Dirac crossing quite well quantitatively, as determined by comparing experimental and theoretical cuts of the spectral function (Fig. $2 \mathrm{C}, \mathrm{D})$ along the energy and momentum directions. The intensity of the plasmaron band in the experimental data is comparable to the theoretical prediction, demonstrating that neither the defect scattering rate nor the symmetry breaking potential induced by the substrate introduce important energy scales. Both of these effects have been predicted to quickly dampen the plasmaron mode $(9,10,26)$.

The doping dependence of the spectral function, presented in Fig. 3, confirms a key prediction of the $G_{0} W$ RPA theory, namely that the spectral function should scale with the Fermi energy $E_{\mathrm{F}}$ (measured with respect to $E_{\mathrm{D}}$ ) and the Fermi momentum $k_{\mathrm{F}}$. This is a special consequence of the linear band structure of graphene and the Coulomb interaction, and follows from the linear scaling of the RPA dielectric function (and therefore the plasmon dispersion) with $E_{\mathrm{F}}$ and $k_{\mathrm{F}}$. (The bare Dirac energy $E_{\mathrm{D}}$ is unknown, so we have equivalently used ( $E_{\mathrm{F}}-$ $E_{0}$ ) to scale the data). These data (Fig. 3A-F) show that over nearly an order of magnitude variation of charge density, the renormalized bands have indistinguishable shape. Furthermore, when cuts of the measured spectral function at $E=E_{1}$ and $k=0$ are plotted with the same scaling, the width $\delta k=\left|k_{+}-k_{-}\right| / k_{\mathrm{F}}=0.38$ and height $\delta E=\mid E_{2}-$ 
$E_{0}|/| E_{\mathrm{F}}-E_{0} \mid=0.48$ of the diamond-shaped region are shown to be constants (Fig. 3G-H) and can therefore be characterized by a single parameter as discussed below. Only at the lowest doping, where the fixed phonon energy scale $\left(\omega_{\mathrm{ph}} \sim 180 \mathrm{meV}\right)$ approaches $\left(E_{\mathrm{F}}-E_{0}\right)$ does any deviation appear, evidence of electron-plasmonphonon mixing.

The parameter which describes the interaction strength is the graphene effective coupling constant $\alpha_{\mathrm{G}}=e^{2} / \in \hbar v_{\mathrm{F}}$ $\sim 2.2 / \epsilon(14)$. (Note that $\alpha_{G}$, an intrinsic parameter of the electron-electron interactions within graphene, is to be distinguished from the vacuum coupling constant $\alpha=e^{2} / \hbar c=1 / 137$ determined by optical studies of graphene that characterizes the free-space Coulomb interaction $(27,28)$.) Here the constant $\epsilon=\left(\epsilon_{\mathrm{a}}+\epsilon_{\mathrm{b}}\right) / 2$ is the average screening contribution of any dielectrics above $\left(\epsilon_{\mathrm{a}}\right)$ and below $\left(\epsilon_{\mathrm{b}}\right)$ the graphene. A series of calculations were performed for different values of $\alpha_{G}$, and from these calculations (shown in supplemental figure S1), values for $\delta E$ and $\delta k v s . \alpha_{\mathrm{G}}$ were extracted (Fig. 3I). Comparing to our measurements, we conclude the best fit is for $\alpha_{\mathrm{G}} \sim 0.5$. From this value, we determine the average screening $\epsilon \sim 4.4$ corresponding to substrate screening contribution $\epsilon_{\mathrm{b}} \sim 7.8$ for graphene on $\mathrm{H}-\mathrm{SiC}$ in vacuum.

The impact of disorder on the plasmaron bands is revealed by the doping dependence of the spectral weight distribution. This is because the dopant ions themselves can act as weak scatterers (17). Fig. 4A,B show magnified views of the Dirac crossing region for two different dopings. We find that at low doping, the intensity of the plasmaron band is stronger than the main band, but at higher doping, the plasmaron band is reduced in strength in accord with predictions $(9,10)$. This is evident in the images in Fig. 4 as well as in energy cuts taken at $k=k$, at one side of the diamond region, (Fig. 4C) and at $k=0$ (Fig. 3H). (This weakening of the plasmaron band is a characteristic of chemical doping and is not expected to appear in samples doped by applied gate voltage.)

Although the plasmaron mode is dampened by defect scattering, the main band dispersion (indicated by the dashed lines in Fig. 4) remains unaltered. So we can expect that even with heavy damping of the plasmaron bands by disorder, the main bands will still be characterized by a kink in the Dirac crossing region. This kink reflects the offset $\Delta E \sim\left(E_{2}-E_{0}\right)$ between upper and lower $\pi$ bands illustrated in Fig. 5D.

In fact, such an energy shift $\Delta E$ is readily observed for ordinary epitaxial graphene samples prepared by annealing $\mathrm{SiC}$ (0001) surfaces in ultrahigh vacuum (29) or in inert atmospheres at high temperature (30), although its interpretation in terms of electronic interactions has been controversial. Ordinary epitaxial graphene samples consist of two carbon layers, an insulating "buffer layer" that saturates the substrate's Si dangling bonds, and a second, decoupled layer which acts as single-layer graphene (31). Such samples have a high doping concentration $\left(n=1.1 \times 10^{13} \mathrm{e} / \mathrm{cm}^{2}\right)$ caused by charge transfer from the substrate, and also have weak satellite bands (17) and rippling $(32,33)$ that arise from the mismatch between the graphene and substrate lattices. There is also a certain degree of disorder at the graphene-SiC interface (34) whose nature is not fully understood.

Comparing the kink at $E_{\mathrm{D}}$ between ordinary graphene (see e.g. Fig. 1(d) of Ref. (17)) to that of quasi-free- 

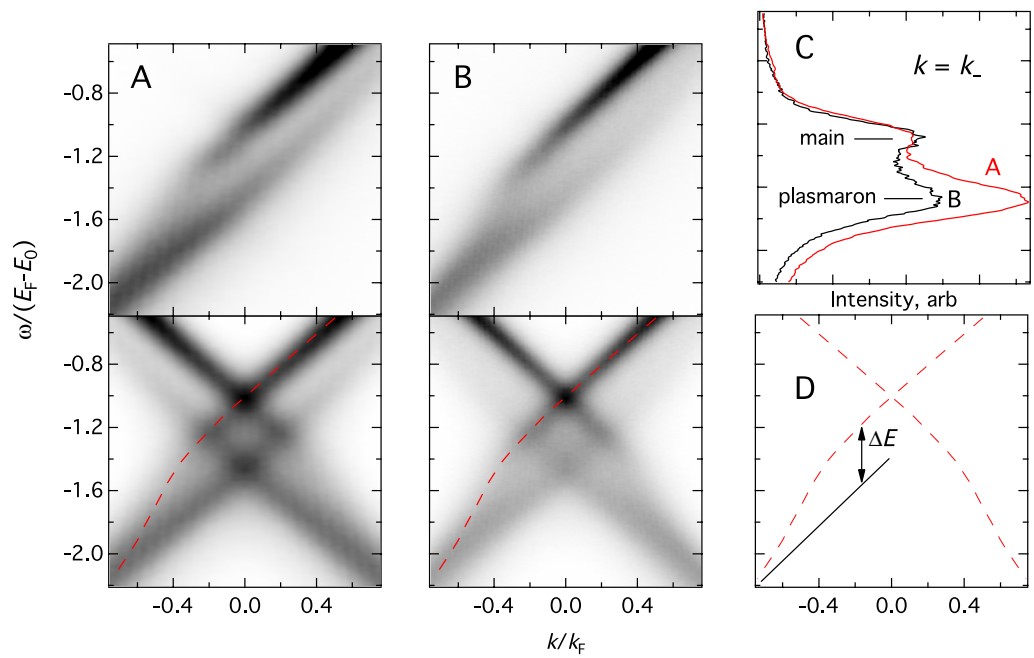

Fig. 4. A-B. Magnified view of the raw spectral function along the $\Gamma K$ direction of the graphene Brillouin zone (upper) and mirror-symmetrized (lower) for two different dopings ( $n=0.7$ and $3.3 \times 10^{13} \mathrm{~cm}^{-2}$, resp.). C Energy spectra at $k=k_{-}$(defined in Fig. 1B) for the same dopings as in parts A-B, scaled to the height of the main peak. Taken together, panels A-C demonstrate the dampening of the plasmaron mode with increased disorder due to chemical doping. D. The dispersion of the main bands is unaltered by doping, indicating that the main band's dispersion function is not sensitive to defect scattering.

standing graphene in Fig. $4 \mathrm{D}$ we find a qualitatively similar but smaller shift $\Delta E$ at a given doping level in the former. From the obvious reduction of $\Delta E$ as $\alpha_{\mathrm{G}} \rightarrow 0$ (Figure $\mathrm{S} 1$ ), we conclude that the effective dielectric constant $\epsilon$ was $\sim 3-4$ times larger in the previous samples than in the quasi-free-standing samples. (This indicates that the interfacial buffer layer and dangling bonds are quite polarizable.) For such high screening, the plasmaron band is not resolvable but appears only as a shoulder on the main band, and it is likely to be dampened by the disorder in those samples.

The quantitative agreement between our measurements for quasi-free-standing samples and the theoretical model is satisfying, but we note some deviations between theory and data in Figs. 1 and 2. At higher binding energies, the model predicts distinct electronic and plasmonic bands, but the experiment shows a merging or, as we suggest, a crossing of these bands. These deviations between the theory and experiment cannot be explained by lattice effects (35), and instead hint at physics beyond that contained in the $G_{0} W$-RPA theory. In this context, graphene can be a good platform for tests of such theories.

For sufficiently high charge density (such that $\left.\left(E_{\mathrm{F}}-E_{\mathrm{D}}\right)>k_{\mathrm{B}} \mathrm{T}\right)$, the equilibrium transport properties will be only modestly affected by the interactions we have seen. This is because transport involves carriers within $k_{\mathrm{B}} T$ of $k_{\mathrm{F}}$, where the plasmaron mode merges to the main band while it is strongly dampened (although not completely suppressed). Nevertheless our results do establish that the dynamics of a hot population of holes injected into $n$-type graphene (or similarly electrons injected into $p$-type graphene) will be subject to the strong plasmon coupling we have observed. These dynamics might be exploited in new devices, such as terahertz amplifiers ( 6 , 7) and stimulated emission of plasmons and plasmon lasing (8).

In the low-doping limit $\left(k_{\mathrm{B}} T>\left[E_{\mathrm{F}}-E_{\mathrm{D}}\right]\right)$, on the other hand, carriers with energy on the Fermi energy scale are thermally excited and should participate in transport (36). In this weakly-doped regime, the RPA theory which assumes that quasiparticle interactions are weak compared to the Fermi energy, is least reliable. As we have shown, the Dirac crossing spectrum is reconstructed in a highly non-trival way even when interactions are weak. Further exploration of this regime to higher temperatures or, equivalently provided that disorder is weak, to lower densities, may reveal more surprises in the future. 
1. D. Bohm, D. Pines, Phys. Rev. 92, 609 (1953).

2. B. Lundqvist, Phys. kondens. Materie 6, 193 (1967).

3. J. L. Shay, W. D. Johnston, E. Buehler, J. H. Wernick, Phys. Rev. Lett. 27, 711 (1971).

4. R. Tediosi, N. P. Armitage, E. Giannini, D. van der Marel, Phys. Rev. Lett. 99, 016406 (2007).

5. O. E. Dial, Single Particle Spectrum of the Two Dimensional Electron Gas, PhD Thesis, MIT (2007).

6. V. Ryzhii, J. Jour. Appl. Phys. 45, L923 (1 Sept 2006, 2006).

7. V. Ryzhii, M. Ryzhii, T. Otsuji, Journal of Applied Physics 101, 083114 (2007).

8. $\quad$ F. Rana, IEEE Transactions on Nanotechnology 7, 91 (2008).

9. $\quad$ E. H. Hwang, S. D. Sarma, Phys. Rev. B 77, 081412 (2008).

10. M. Polini et al., Phys. Rev. B 77, 081411 (2008).

11. D. P. DiVincenzo, E. J. Mele, Phys. Rev. B 29, 1685 (1984).

12. T. Ando, T. Nakanishi, J. Phys. Soc. Jpn. 67, 1704 (May 1998, 1998).

13. T. Ando, T. Nakanishi, R. Saito, J. Phys. Soc. Jpn. 67, 2857 (Aug 1998, 1998).

14. A. H. C. Neto, F. Guinea, N. M. R. Peres, K. S. Novoselov, A. K. Geim, Reviews of Modern Physics 81, 109 (2009).

15. C. Riedl, C. Coletti, T. Iwasaki, A. A. Zakharov, U. Starke, cond-mat/0911.1953, (2009).

16. F. Speck et al., paper presented at the 13th International Conference on Silicon Carbide and Related Materials, Nuremberg (Germany), 2009.

17. A. Bostwick, T. Ohta, T. Seyller, K. Horn, E. Rotenberg, Nat. Phys. 3, 36 (2007).

18. A. Bostwick et al., New J. Phys 9, 385 (2007).

19. M. Mucha-Kruczynski et al., Phys. Rev. B 77, 195403 (2008).

20. B. J. Kim et al., Nat. Phys. 2, 307 (June 2006, 2006).

21. L. Perfetti et al., Physical Review Letters 87, 216404 (2001).

22. A. Sekiyama et al., Nature 403, 396 (2000).

23. P. von Allmen, Phys. Rev. B 46, 13345 (1992).

24. E. H. Hwang, S. Das Sarma, Phys. Rev. B 75, 205418 (11 May 2007, 2006).

25. Y. Liu, R. F. Willis, K. V. Emtsev, T. Seyller, Phys. Rev. B 78, 201403 (2008).

26. A. Qaiumzadeh, R. Asgari, New J. Phys. 11, 095023 (2009).

27. R. R. Nair et al., Science 320, 1308 (2008).

28. A. B. Kuzmenko, E. van Heumen, F. Carbone, D. van der Marel, Phys. Rev. Lett. 100, 117401 (2008).

29. I. Forbeaux, J. M. Themlin, J. M. Debever, Phys. Rev. B 58, 16396 (1998).

30. K. V. Emtsev et al., Nat. Mater. 8, 203 (2009).

31. K. V. Emtsev, F. Speck, T. Seyller, L. Ley, J. D. Riley, Phys. Rev. B 77, 155303 (2008).

32. S. Kim, J. Ihm, H. J. Choi, Y.-W. Son, Phys. Rev. Lett. 100, 176802 (2008).

33. F. Varchon, P. Mallet, J. Y. Veuillen, L. Magaud, Phys. Rev. B 77, 235412 (2008).

34. V. Brar et al., Appl. Phys. Lett. 91, 122102 (2007).

35. A. Hill, S. A. Mikhalov, K. Zeigler, Europhys. Lett. 87, 27005 (2009).

36. O. Vafek, Phys. Rev. Lett. 97, 266406 (2006).

Acknowledgements. The Advanced Light Source is supported by the Director, Office of Science, Office of Basic Energy Sciences, of the U.S. Department of Energy under Contract No. DE-AC02-05CH11231. Work in Erlangen was supported by the German Research Council (DFG) through research grant SE 1087/5-1 and through the Cluster of Excellence 'Engineering of Advanced Materials' at the University of ErlangenNuremberg. AHM acknowledges support from the Welch foundation and CERA. 


\section{Supplementary Information}
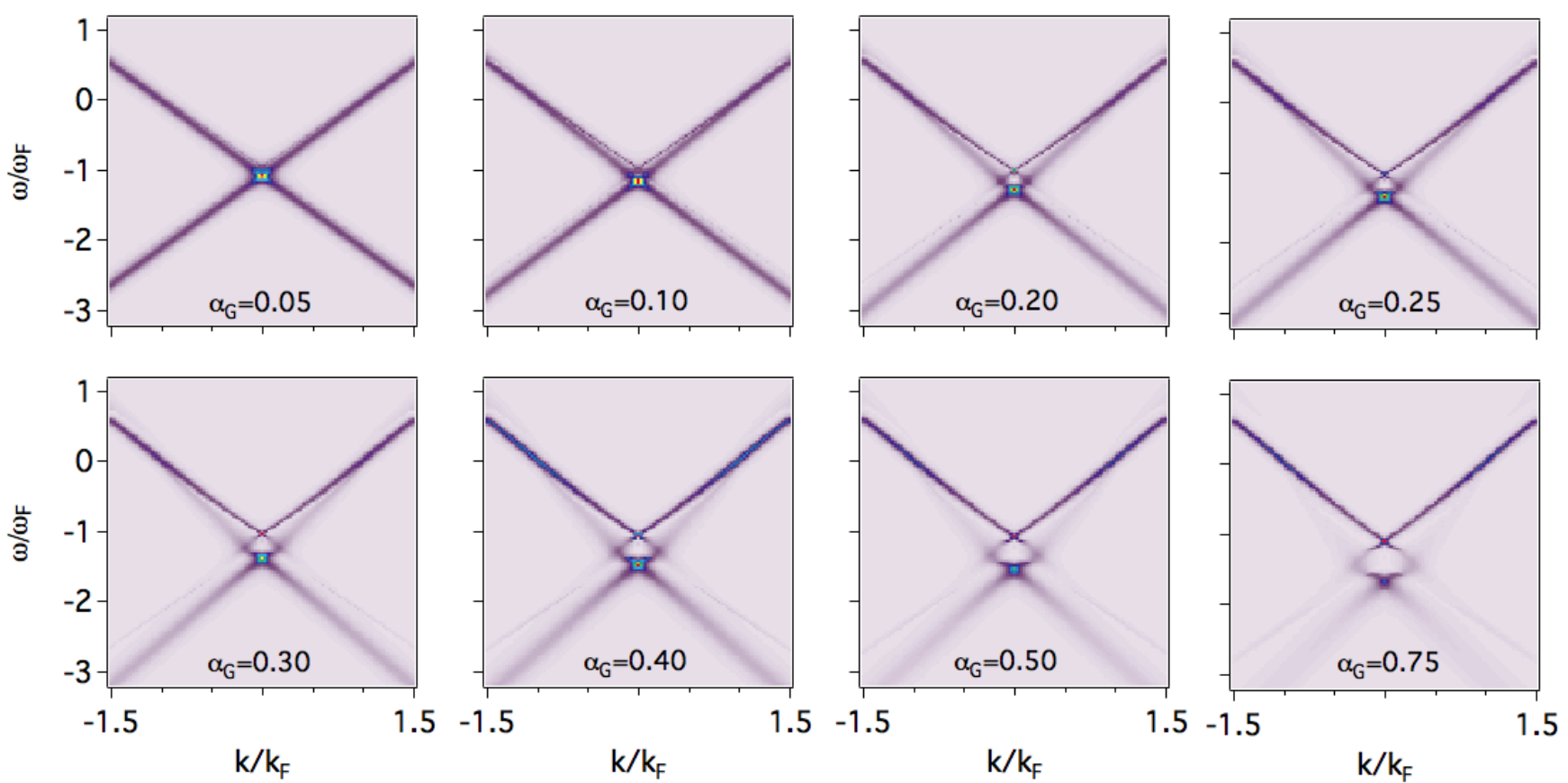

Figure S1. Calculated spectral functions for doped graphene, as a function of the coupling constant $\alpha_{\mathrm{G}}$ as indicated. Spectra are broadened to approximate modest energy and momentum resolution by adding a background contribution of $0.01 \omega_{\mathrm{F}}$ to $\operatorname{Im} \Sigma$. 